\title{
Supracutaneous plating in tibial metadiaphyseal fractures with compromised soft tissue using LCP as an external fixator
}

\author{
Kalia S. ${ }^{1}$, Sharma S. ${ }^{2}$, Sehgal M. ${ }^{3}$, Kanwar S. ${ }^{4}$ \\ ${ }^{1}$ Dr. Sandeep Kalia, Assistant Professor, Orthopaedics, ${ }^{2}$ Dr. Shalini Sharma, Assistant Professor, Anaesthesia, ${ }^{3}$ Dr. Manik \\ Sehgal, Senior Resident, Orthopaedics, ${ }^{4}$ Dr. Sachin Kanwar, Orthopaedics, Regional Hospital; all authors are affiliated \\ with DRPGMC, Tandakangra (H.P.) India.
}

Corresponding Author: Dr. Shalini Sharma, Assistant Professor, Anaesthesia, DRPGMC, Tandakangra (H.P.) India. E-mail: shalini.medico@gmail.com

\begin{abstract}
Introduction: Tibial meta-diaphysealfractures are challenging injuries because of subcutaneous anteromedial surface of bone and frequent soft tissue complications. Ideal management requires stable fixation with minimal soft tissue handling. Locking compression plate (LCP) applied as an external fixator fulfills these criteria and can be an important tool in the armamentarium of an orthopaedic surgeon. Objective of the study is to evaluate the result of this technique. Methods: 23 patients with meta-diaphyseal fractures of tibia with compromised soft tissue treated by this technique were evaluated retrospectively interms of union, maintenance of reduction, complications and final outcome using KSS (Knee society scoring) for proximal fractures and AOFAS (AO Ankle and Foot scoring) for distal fractures. Average follow up duration was 9.4 months (6-14 months). Results: There were fifteen males, eight females with fourteen open fractures and nine cases of closed fractures with tscherne grade 2 or 3 soft tissue injury. Five fractures were in proximal tibia and eighteen were in distal tibia, all fractures united with an average of 21.7 weeks period maintaining acceptable reduction. There were 3 cases of infection, one deep and two superficial treated successfully by debridement and antibiotics. Average knee society scores were 74 and 78.6 for open and close proximal metadiaphyseal fractures respectively. Average AOFAS scores were 81.9 and 84 for open and close distal tibial metadiaphyseal fractures respectively. Conclusion: we found this technique to be more biological, simple to do surgery, effective in maintaining reduction till union, more patient friendly in comparison to traditional large, bulkyfixators and with minimal complications.
\end{abstract}

Keywords: subcutaneous anteromedial surface, LCP, tibial metadiaphyseal fractures

\section{Introduction}

Tibial fractures pose a challenge to treating orthopaedic surgeon because this bone is subcutaneous on its anteromedial surface and has weight bearing joints on its both ends. This peculiarity demands maximal anatomical reduction and diligent soft tissue care so as to achieve early union with maximum function. Surgery is the acceptable method for treating tibial fractures [1].

However because of subcutaneous anteromedial surface and high velocity trauma, the surgery had frequent complications of skin break down and infection in open reduction and internal fixation [2,3]. Hence the evolution of surgical techniques was to stabilize these fractures with minimal invasiveness to maintain the

Manuscript Received: $10^{\text {th }}$ April 2019

Reviewed: $20^{\text {th }}$ April 2019

Author Corrected: $26^{\text {th }}$ April 2019

Accepted for Publication: $30^{\text {th }}$ April 2019 biology of soft tissue and bone [4,5]. Tibial diaphyseal fractures could easily be treated with closed interlocking nails but metaphyseal areas at both ends had surgical dilemma in choosing the implant and technique so as not to violate already traumatized soft tissue $[6,7]$. MIPO technique and low profile anatomical locking compression plating reduced the risk of skin breakage and infection to some extent with promising results [8].

However still some of the studies showed unacceptably high incidence of implant prominence (52\%) and late infection upto $14 \%$ [9]. Standard external fixators and ring fixators maintained the biology but were bulky and cumbersome to patients owing to size. A new technique in armamentarium to manage these fractures was supracutaneous plating $[10,11]$. The use of this 
technique was reported in literature as early as 1991 but for two decades there were only sporadic reports with small number of patients series [10]. In this technique the anatomical contoured LCP with angular stable screw is applied as an external fixator supracutaneously which maintains the stabilization without violating the biology of soft tissue.

This technique is simple, biological and is patient friendly in terms of being low profile external fixator, no radiological silhouette, and ease of implant removal in out patient set up.

Purpose of this study was to retrospectively evaluate the results of using LCP as an external fixator at our institute.

\section{Methods}

This was a retrospective cohort study conducted in the department of Orthpaedics in a Medical College of Northwest India. For the purpose of the study, records of all patients admitted in the orthopaedics ward from March 2014 to March 2016 were retrieved, after taking due ethical clearance from the ethical committee of the medical college.

Inclusion Criteria- All patients who were admitted during the above mentioned time period, with metadiaphyseal tibial fractures having compromised soft tissue (Tscherene grade 2 and 3 ) as well as all metadiaphyseal open tibial fractures were included in the study.

Exclusion Criteria- Those patients with diaphyseal tibial fractures, tibial fractures in paediatric age groups and pathological fractures were excluded from the study.

The total number of patients fulfilling the above criteria and had been admitted to the orthopaedics ward during above mentioned period were 23. All the patients had been operated upon using anatomical contoured LCP as an external fixator.

\section{Original Research Article}

Surgical Technique- All patients were operated on radiolucent table under regional or general anesthesia. Open fractures were converted to close fractures after debridement and closure. Fractures were reduced indirectly with traction and acceptable anatomical reduction was ascertained on fluoroscopy. Reduction was maintained with percutaneous clamps or temporary $\mathrm{k}$ wires. Anatomical low profile LCP of adequate size was placed on subcutaneous anteromedial surface of tibia and position checked on $\mathrm{C}$-arm. After ascertaining the proper position on $\mathrm{C}$-arm, the plate was held with $\mathrm{k}$ wires at both ends.

Then the plate was raised with folded towel for about 20 mm over the skin and locking screws were applied after drilling through the plate holes. Ideally both cortical purchase was done and minimum of four to five screw were applied on either side of fracture. Final position and reduction checked on $\mathrm{C}$-arm and anti septic dressing applied.

Post Operative Protocol- Pin tract dressing was done with saline daily and care was taught to patient. Non weight bearing crutch walking was allowed as soon as comfortable to patient. Active and assisted joint range of motion was started from the first post operative day.

The patients were then followed up every 6 weeks till the removal of fixator.At each visit clinico- radiological assessment for union, range of motion and any new complication was assessed. Progressive weight bearing was allowed according to clinic-radiological assessment of fracture union status. Plate removal was done in out patient set up once the patient was fully weight bearing without the pain at fracture site and $\mathrm{X}$ rays showed healing of fracture.

At final follow up the knee society scoring (KSS) and AO ankle and Foot scoring (AOFAS) was done for proximal tibial fractures and distal tibial fractures respectively. Both AOFAS and KSS scores were calculated using free online scoring calculator available on ortho toolkit)

\section{Results}

A total of 23 patients ( 8 females 15 males) with age range of 15 years to 76 years were included in the study. There were 18 cases of distal tibial fractures and 5 cases of proximal tibial fractures. There were 14 cases of open fractures $(2$ cases of grade 1, 1 case of grade $3 \mathrm{~A}$ and 11 cases of grade 2 open on gustilo Anderson classification). Nine cases were of closed injuries with compromised soft tissue as grade 2, grade 3 on Tscherne classification with echymosis and blistering on skin including one case of impending compartment syndrome which was treated with fasciotomy and stabilization with supra cutaneous plating (Table 1). Three patients had associated injuries of fracture shaft femur, midfoot (lisfranc injury) and comminuted patella fracture. Minimum post operative follow up was six months and maximum14 months with average of 9.4 months. 
Original Research Article

Table-1: Grading according to soft tissue injury.

\begin{tabular}{|c|c|c|c|c|c|c|}
\hline \multirow{2}{*}{} & \multicolumn{2}{|c|}{ Gustilo anderson classification } & \multicolumn{2}{c|}{ Tscherne classification } \\
\cline { 2 - 6 } & Grade 1 & Grade 11 & Grade 111 & Grade 11 & Grade 111 \\
\hline \multicolumn{2}{|c|}{ Sex (M/F) } & $1 / 1$ & $6 / 5$ & $1 / 0$ & $6 / 2$ & $1 / 0$ \\
\hline \multirow{2}{*}{ Tibia Fracture } & Proximal & - & 2 & - & 3 & - \\
\cline { 2 - 7 } & Distal & 2 & 9 & 1 & 5 & 1 \\
\hline
\end{tabular}

Fracture union occurred in all cases with earliest at 12 weeks and latest at 24 weeks, average 21.7 weeks. In three cases of delayed fracture healing bone marrow injection was done. On follow up, there was a reported complication in one case with deep infection at $14^{\text {th }}$ post operative day which was successfully treated with debridement and antibiotic beads and there were two cases with screw site drainage which settled with dressing and oral antibiotics for two weeks. In one case during plate removal the screw was jammed and was cut with a cutter to remove the plate. One case in series had a post operative fall resulting in bending of the plate and angulating the fracture. Reduction was done with plate benders under sedation and monitored anaesthesia care in Operation Theater without revising the implant. Fig 1,2,3

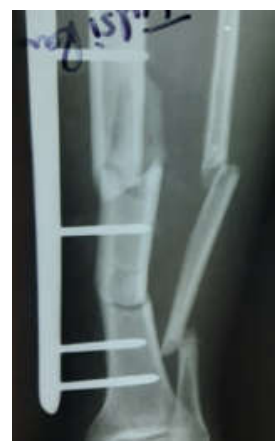

Fig. 1

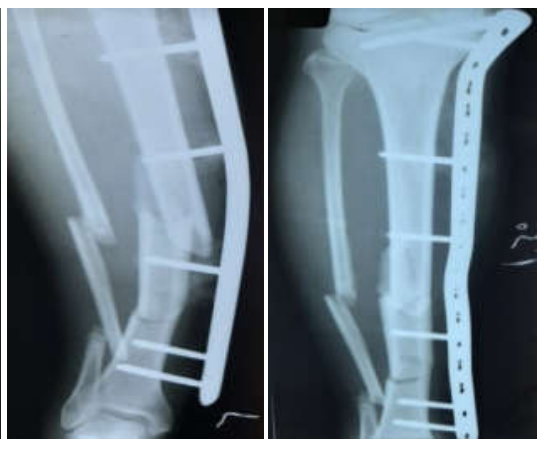

Fig. 2

Fig. 3

Fig. 1-Supracutaneous plate in segmental tibia fracture

Fig 2- Plate bent due to fall

Fig 3- In situ correction of reduction with plate bender

Functional outcome was good in all patients with average ankle dorsiflexion of 20 degree and plantar flexion of 30 degree and average knee range of motion 0 to 130 degree. AOFAS average score was 83.5 with minimum of 77 and maximum of 90 in patients with distal tibia fractures.

Table-2: Outcome according to fracture site and soft tissue injury.

\begin{tabular}{|l|c|c|c|c|}
\hline \multirow{2}{*}{} & \multicolumn{2}{|c|}{ Proximal tibia } & \multicolumn{2}{c|}{ Distal tibia } \\
\cline { 2 - 5 } & Open fracture & Closed fracture & Open Fracture & Closed fracture \\
\hline Number of Patients (n) & 2 & 3 & 12 & 6 \\
\hline $\mathrm{M} / \mathrm{F}$ & $1 / 1$ & $3 / 0$ & $7 / 5$ & $4 / 2$ \\
\hline Age (years) & 45.5 & 53.3 & 49.2 & 39.8 \\
\hline Union (weeks) & 20.6 & 18.4 & 23.6 & 22.4 \\
\hline Scores (KSS)* & 74 & 78.6 & - & - \\
\hline Scores (AOFAS) $\dagger$ & - & - & 81.9 & 84 \\
\hline
\end{tabular}

*KSS -Knee society score; †AOFAS-American orthopaedic association foot and ankle score.

Knee society score average was 76.8 with minimum of 67 and maximum of 82 in proximal tibia fractures. According to KSS (knee society score) in five cases of proximal tibia, two were rated excellent, two cases as good and one case as fair. (Table 2) The reason for low score in this patient was associated fracture of patella in which inferior pole patellectomy and patellar tendon repair was done. 


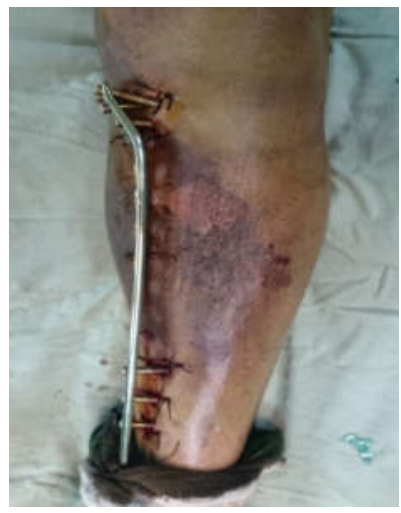

Fig-4: Clinical picture of supracutaneous plate

\section{Discussion}

The Concept of extracorporeal plating to stabilize the fracture is not new. Mark R K and Van Der C used standard A O plates for severe open long bone fractures and pseudo arthrosis in 12 cases as early as 1991 with successful outcome [10]. They used nut on the under surface to stabilize the plate extra corporeal. After the invent of locking plates with angular stable screw, Kloen described the use of LCP as a low profile external fixator and coined the term Supracutaneous plating [11]. For almost a decade there were sporadic studies on this technique. Sven A F and Simon D in 2012 used the LCP as a low profile external fixator in patients with infected non union of disal tibial metaphyseal area and reported good results in a series of seven patients [12].

Acase series of five patients by Gupta SV and Parimala SP, fourteen patients by Qui XS et al, ten cases by Panda SS and Panda D and thirty patients by Srinivas Prabhu and Binayak Ray all showed good results with the use of LCP as an external fixator in tibial metadiaphyseal fractures with compromised soft tissue. $[13,14,15,16]$. Zhang J and Ebrahim NA even extended the use of externalized LCP in closed fractures without compromised soft tissue and reported successful use of LCP as an external fixator in both open and closed tibial metaphyseal fractures $[17,18]$. They however used femoral LCP instead of tibial LCP in their patients owing to better stiffness of femoral LCP over tibial LCP. Biomechanically axial and torsional strength of externalized LCP has been an issue of concern and questioned in few studies.

Ahmad $\mathrm{M}$ et al in their study found that strength and construct stability significantly reduces when plate bone distance is more than $2 \mathrm{~mm}$ [19]. Zhang J, Ebrahim $\mathrm{N}$ in their study concluded that for stable external fixation with LCP, the plate bone distance should be less than
$30 \mathrm{~mm}$ [20]. Wei Lu, Li Hui in their study of comparison of axial and torsional stiffness of LCP found femoral LCP to be significantly better than tibial LCP [21]. Kanchanamoi C and Phiphohomegkol N studied the endurance of externalized LCP in tibial fracture by applying 500,000 times cyclic loading to construct which is approximately 6 months of weight bearing. There was no construct failure. They concluded that failure of LCP as an external fixator is unlikely to be a critical issue [22].

Comparison of axial and torsional stiffness of externalized LCP and standard unilateral external fixator was studied by the Ang B H, Chen J Y et al and they found that mean axial stiffness was comparable between two and torsional stiffness was statistically higher in externalized LCP than standard unilateral external fixator [23]. Systematic review of literature on supracutaneous plating also concluded that though internal fixation by LCP is stronger than externalized LCP but there was no significant difference between externalized LCP and traditional external fixator in terms of biomechanics $[7,24]$.

In our patient series of 23 patients we also found this to bean effective method with good results in patients with compromised soft tissue. All fractures achieved union eventually maintaining acceptable reduction. Only four cases (19.04\%) required additional procedure. Debridement for deep infection in one case and bone marrow injections for delayed healing in three patients. We had used tibial LCP instead of more stiff femoral LCP, but there was no implant breakage or failure.

Reason for this could be delayed weight bearing in our patients till the clinic-radiological evidence of fracture healing. This low profile external fixation is more biological and is patient friendly as could easily be 
concealed in clothing and did not hamper with the gait and activities of daily living of patient. There was no radiological silhouette with supracutaneous plating and the removal of plate was also easy in the out patient set up. Post operative improvement of mediolateral angulation and to some extent of rotational correction can be done with plate benders with plate in situ.

Dynamization is possible with this technique by removing screws closer to fracture site as the plate acts as a load sharing device. Limitations with this technique is that prior acceptable anatomical reduction is necessary before applying the plate. Also the longer plates with longer screws are required which should be specifically available in implant tray and need to be checked beforehand.

The above mentioned limitations do not deter a surgeon from using the supracutaneous LCP as it continues to be a better technique, less cumbersome and with better results.

However this technique is less adopted as compared to the standard technique of internal fixation in India. Therefore the general practice needs to be reviewed with further studies on this technique.

\section{Conclusion}

This technique of supracutaneous plating by applying low profile anatomical LCP plate as a monolateral external fixator for definitive treatment is effective in patients with compromised soft tissue being more biological, easy to carry out surgery, more patient friendly and with minimal complications.

However a prospective randomised double blind study is required to compare this with MIPO, and standard unilateral external fixator.

\section{Contribution by authors}

- Sandeep Kalia: Conceived the study, designed and developed the methodology and performed the study.

- Shalini Sharma: Writing of the manuscript and proofreading.

- Manik Sehgal: Assisted in surgery, collected the data and helped in data analysis.

- Sachin Kanwar: Assisted in surgery, collected the data and helped in data analysis.

Conflict of interest: None declared. Funding: Nil, Permission from IRB: Yes

\section{Original Research Article}

\section{References}

1. Jensen JS, Hansen FW, Johansen J. Tibial shaft fractures. A comparison of conservative treatment and internal fixation with conventional plates or $\mathrm{AO}$ compression plates. Acta Orthop Scand. 1977;48(2): 204-12.

2. Young MJ, Barrack RL. Complications of internal fixation of tibial plateau fractures. Orthop Rev. 1994 Feb; 23 (2):149-54.

3. Philip A. McCann, Jackson $\mathrm{M}$, Steve $\mathrm{T}$ et al. Complication of definitive open reduction and internal fixation in tibial pilon fractures. Intl. Orthop2011 Mar; 35 (3): 413-418.

4. Perren SM. Evolution of the internal fixation of long bone fractures. The scientific basis of biological internal fixation: choosing a new balance between stability and biology. J Bone Joint Surg Br. 2002 Nov; 84 (8): 1093110.

5. Borrelli J Jr, Prickett W, Song E, et al. Extraosseous blood supply of the tibia and the effects of different plating techniques: a human cadaveric study. J Orthop Trauma. 2002 Nov-Dec;16(10):691-5.

6. McCann PA, Jackson M, Mitchell ST, et al. Complications of definitive open reduction and internal fixation of pilon fractures of the distal tibia. Int Orthop. 2011 Mar;35(3):413-8. doi: 10.1007/s00264010-1005-9. Epub 2010 Mar 30.

7. Luo $\mathrm{P}, \mathrm{Xu} \mathrm{D}, \mathrm{Wu} \mathrm{J}$, et al. Locked plating as an external fixator in treating tibial fractures: A PRISMAcompliant systematic review. Medicine (Baltimore). 2017 Dec; 96 (49):e9083. doi: 10.1097/MD. 000000000 0009083 .

8. Gupta $\mathrm{P}^{1}$, Tiwari $\mathrm{A}^{1}$, Thora $\mathrm{A}$, et al. Minimally Invasive Plate Osteosynthesis (MIPO) for Proximal and Distal Fractures of The Tibia: A Biological Approach. Malays Orthop J. 2016 Mar;10(1):29-37. doi: 10.5704/ MOJ.1603.006.

9. Lau TW, Leung F, Chan CF, et al. Wound complication of minimally invasive plate osteosynthesis in distal tibia fractures.x Int Orthop. 2008 Oct;32(5): 697-703. Epub 2007 Jun 16.

10. Marti RK, van der Werken C. he AO-plate for external fixation in 12 cases. Acta Orthop Scand. 1991 Feb;62(1):60-2. 


\section{Original Research Article}

11. Kloen P. Supercutaneous plating: use of a locking compression plate as an external fixator. J Orthop Trauma. 2009 Jan; 23(1): 72-5. doi: 10.1097/BOT. 0b 013e 31818f8de4.

12. Tulner SA, Strackee SD, Kloen P. et al. Metaphyseal locking compression plate as an external fixator for the distal tibia. Int Orthop. 2012 Sep;36(9): 1923-7. doi: 10.1007/s00264-012-1585-7. Epub 2012 Jun 1.

13. Gupta SKV, Parimala SP. Supracutaneous Locking Compression Plate for Grade I \& II Compound Fracture Distal Tibia- A Case Series Open Journal of Orthopedics. 2013, 3, 106-109

14. Qiu XS, Yuan H, Zheng X, et al. Locking plate as a definitive external fixator for treating tibial fractures with compromised soft tissue envelop. Arch Orthop Trauma Surg. 2014 Mar;134 (3): 383-8. doi: 10.1007/ s00402-013-1916-1. Epub 2013 Dec 22.

15. Panda SS, Panda D, Suri N. Supracutaneous plating -use of LCP as an external fixator for intra articular compound fractures. International Journal of Medical Research \& Health Sciences, 2016, 5, 8:62-67

16. Shrinivas P, Binayak R. A study of Locking compression plate as external fixator in the definitive management of open diaphyseal fractures of tibia in adults. IOSR Journal of Dental and Medical Sciences 2019 Jan; 18(1):7-13

17. Zhang J, Ebraheim N, Li M, et al. External fixation using femoral less invasive stabilization system plate in tibial proximal metaphyseal fracture. Clin Orthop Surg. 2015 Mar;7(1):8-14. doi: 10.4055/cios.2015.7.1.8. Epub 2015 Feb 10.
18. Zhang J, Ebraheim NA, Li M, et al. External Fixation Using a Locking Plate: A Reliable Way in Treating Distal Tibial Fractures. J Orthop Trauma. 2015 Nov; 29(11): e454-8. doi: 10.1097/BOT. 00000 00000000377.

19.AhmadM, Nanda R, Bajwa AS, et al. Biomechanical testing of the locking compression plate: when does the distance between bone and implant significantly reduce construct stability? Injury. 2007 Mar; 38(3):358-64. Epub 2007 Feb 12.

20. Zhang J, Ebraheim N, Li M, et al. External fixation using locking plate in distal tibial fracture: a finite element analysis. Eur J Orthop Surg Traumatol. 2015 Aug;25(6):1099-104. doi: 10.1007/s00590-015-1604-7. Epub 2015 Feb 21.

21. Wei Liu, Lihui Y, Xiaochuan K et al. Stiffness of the locking compression plate as an external fixator for treating distal tibial fractures: a biomechanics study. BMC Musculoskelet Disord. 2017; 18: 26.

22. KanchanomaiC, Phiphobmongkol V.Biomechanical evaluation of fractured tibia externally fixed with an LCP. J Appl Biomech. 2012 Nov;28(5): 587-92. Epub 2012 May 9.

23. Ang BFH, Chen JY, Yew AKS, et al. Externalised locking compression plate as an alternative to the unilateral external fixator: a biomechanical comparative study of axial and torsional stiffness. Bone Joint Res. 2017 Apr;6(4):216-223. doi: 10.1302/2046-3758. 64. 2000470.

24. Win MT, Yasser M, Tint L et al. Supracutaneous plate: A comparable alternative to external fixator in the treatment of open fracture. IJOS 2018; 4(2): 959-962.

\section{How to cite this article?}

Kalia S, Sharma S, Sehgal M., Kanwar S. Supracutaneous plating in tibial metadiaphyseal fractures with compromised soft tissue using LCP as an external fixator. Surgical Update: Int J surg Orthopedics. 2019;5(2):110-115.doi:10.17511/ ijoso.2019.i02.08. 